\title{
The treatment of cubitus valgus using the ilizarov method
}

\begin{abstract}
Cubitus valgus is the most common complication of lateral condylar fractures. Various combinations of osteotomy and fixation have been described to correct valgus deformity. We are using distraction osteogenesis with Ilizarov technique to treat 11 elbows in 11 patients with cubitus valgus. The clinical outcome was evaluated using the protocal of Bellemore et al. ${ }^{1}$ The mean time to follow up was 20 months (15 to 35 ) and the mean time to Ilizarov frame removal was 14 weeks (10 to 18$)$. The mean carrying angle was corrected from $35^{\circ}\left(25^{\circ}\right.$ to $\left.40^{\circ}\right)$ to $10.4^{\circ}\left(5^{\circ}\right.$ to $\left.15^{\circ}\right)$ in patients with cubitus valgus. There were 8 excellent and 3 good results.
\end{abstract}

Keywords: Cubitus valgus, Ilizarov, Osteotomy
Volume 3 Issue 3 - 2015

\author{
Bari MM,' Shahidul Islam, ${ }^{2} \mathrm{NH}$ Shetu, ${ }^{2}$ \\ Mahfuzer Rahman, ${ }^{2}$ Masum Billah $\mathrm{Md}^{2}$ \\ 'Chief Consultant, Bari-llizarov Orthopaedic Centre,Visiting and \\ Honored Prof., Russian IlizarovScientific Centre, Russia \\ ${ }^{2}$ Bari-llizarov Orthopaedic Centre, Bangladesh
}

Correspondence: Mofakhkharul Bari, Chief Consultant, BariIlizarov Orthopaedic Centre,Visiting and Honored Professor, Russian Ilizarov Scientific Centre, Kurgan, Tel +88 01819211595 , Email bari.illizarov3।@gmail.com

Received:September 03, 2014 | Published: September I5, 2015

\section{Introduction}

The Ilizarov technique with gradual controlled coordinated stretching is a safe and versatile method of treating cubitus valgus deformity at the elbow without the problems of an unsightly scar or limited range of movement and gives a good clinical and radiological outcome.

Fracture of the lateral condyle is the most common fractures of the elbow in children. ${ }^{2-4}$ In cubitus valgus malunion is very common.

Most of the operations are associated with technical problems and complications. ${ }^{5}$ These include under or overcorrection, neuropraxia, an unsightly scar, refracture at the osteotomy site, limitation of range of movement at the elbow, infection and nerve injury.

We have used distraction osteogenesis by Ilizarov technique for the treatment of patients with cubitus valgus. ${ }^{6}$ It differs from the others by applying the principles of deformity correction described by Ilizarov. ${ }^{6}$

\section{Materials and methods}

We treated 11 elbows in 11 patients by osteotomy and gradual controlled coordinated stretching using the Ilizarov method. There were 8 boys and 3 girls with a mean age of 13 years ( 7 to 16). Cubitus valgus occurred after a fracture of the lateral condyle in 11 patients. Four patients with cubitus valgus had a tardy ulnar nerve palsy. We did not do any ulnar transposition of the nerve but we observed surprisingly that gradual distraction relieved the pain due to neohistogenesis.

\section{Surgical technique}

The surgical intervention was carried out with the patient supine and the affected arm on a arm table. We did the surgery without tourniquet.

The pre-operative planning is carried out as follows: at first, the joint line is marked on the radiograph. Next a line is drawn at an angle of $6^{\circ}$ to $8^{\circ}$ to establish the normal valgus angle of the elbow (the valgus inclination line/angle). A line perpendicular to thus is constructed (the distal vertical line) which crosses the anatomical axis of the humerus at the centre of rotation of angulation (CORA). A line bisecting the obtuse angle of this intersect represents the line of osteotomy.

The first (distal reference) wire is inserted from the posterolateral aspect of the lateral condyle and is passed to the anterior cortex of the medial epicondyle at $6^{\circ}$ to $8^{\circ}$ valgus inclination to the joint line. The second wire is inserted from the posteromedial aspect of the medial epicondyle and passed to the anterior cortex of the lateral condyle. These two wires should be olive-tipped to avoid translation of the distal half circle. A second full circle is then applied approximately $4.0 \mathrm{~cm}$ to $5.0 \mathrm{~cm}$ above the apex of the deformity. A motor unit is then placed on the concave aspect of the frame at $90^{\circ}$ to the axis of the hinges. The hinge at the line of osteotomy on the convex side of the deformity is aligned with the centre of rotation of angulation. An opening wedge correction is achieved without any translation, shortening or lengthening (Figure 1a-1c). A third full circle can be applied as necessary above the second in the same manner. All the wires are inserted under image intensification. After applying the frame, small incision is made over the apex of the deformity.

After carrying out the osteotomy, all the clamps and nuts are retightened and the final position checked with the image intensifier.

Post-operatively, all wire sites are cleaned meticulously with antiseptics.
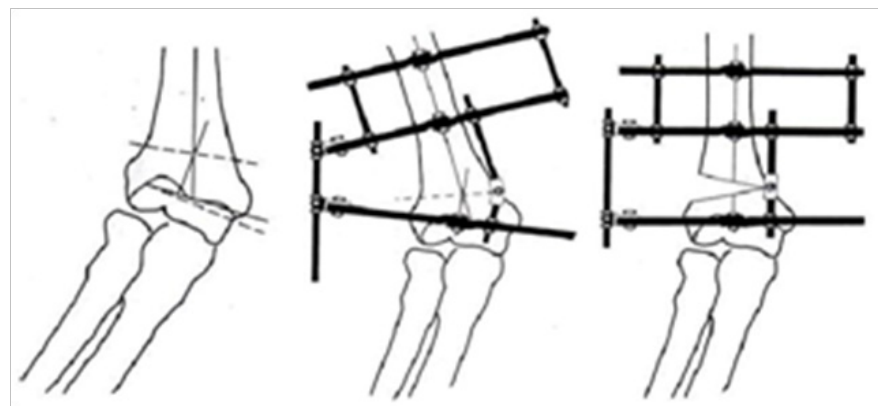

Figure I Diagrams showing the llizarov method of fixation for cubitus valgus. 
a) The pre-operative plan. The most distal dotted line is the joint line. The valgus inclination line is set at 60 to 80 to the joint line in the coronal plane. The line perpendicular to this is the axis of the humerus of the centre of rotation of angulation. The upper dotted line bisects the obtuse angle of this intersect and represents the line of osteotomy.

b) The humeral frame. The lower humeral angle formed is at the level of the valgus inclination line, and the upper humeral ring at the level of the middle third of the diaphysis.

c) The hinge is at the level of the bisector line and lies over the medial bisector line osteotomy opened cortex around the centre of rotation of angulation, thereby correcting the deformity.

Elbow and shoulder exercises were started on the first postoperative day. Distraction was started after five days and took place four times a day $(0.25 \times 4=1 \mathrm{~mm}$ per day). Distraction was continued until full correction of the deformity had been achieved. The results were evaluated according to the system of Barrats et al. ${ }^{1}$ A carrying angle of $\leq 5^{\circ}$ and limitation of flexion and extension by $\leq 10^{\circ}$ was considered on excellent outcome. A carrying angle of $6^{\circ}$ to $10^{\circ}$ and limitation of flexion and extension by $\leq 20^{\circ}$ was considered a good outcome and a carrying angle of $>10^{\circ}$ and loss of flexion and extension of $\geq 20^{\circ}$ was considered to be a poor outcome.

\section{Results}

The mean period of follow up was 20 months (15-40), the mean time to removal of Ilizarov from was 14 weeks (10-16 months).

All patients had an excellent (8 elbows) or good (3 elbows) outcome. The mean preoperative carrying angle of the patients with cubitus valgus was $35^{\circ}\left(25^{\circ}-40^{\circ}\right)$. The mean carrying angle at the last follow up was $8^{\circ}\left(3^{\circ}-13^{\circ}\right)$. The four patients with tardy ulnar nerve palsies, who did not undergo a transposition procedure, relieved pain with the successful correction of the deformity through distraction histoneogenesis: All minimal superficial pin tract infection were treated with oral antibiotics and proper dressings without any problems. Figure 2: Case 1.
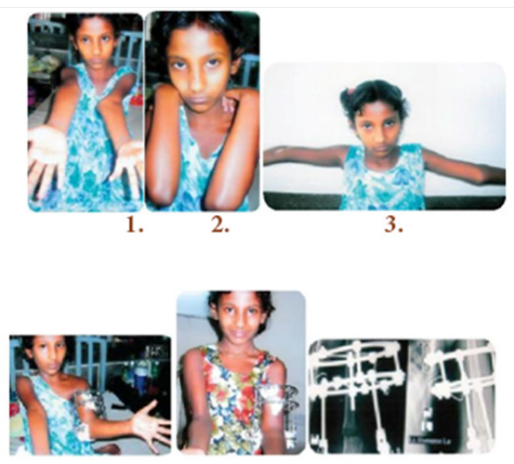

4. 56.

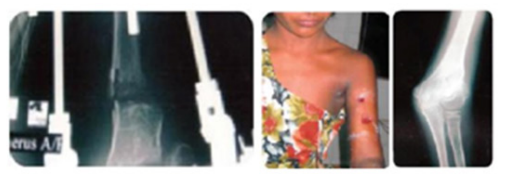

7. 8.

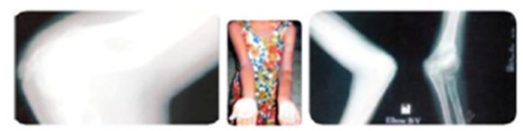

10.

11

12.
Figure 2 Case I

I. 9 years old girl, nonunion lateral condyle, left cubitus valgus with tardy ulnar nerve palsy.

2. Left deformed elbow.

3. Left cubitus valgus 9 years old girl, elbow in extension.

4. One month after osteosynthesis with Ilizarov (opening wedge valgus osteotomy).

5.After two months post. op.

6. Radiographic view of left elbow with Ilizarov in situ after one month follow up.

7. Radiograph of lateral condyle fracture with cubital valgus and open wedge osteotomy of left humerus.

8. Just after removal of the llizarov apparatus.

9. Radiographic union of left lateral condyle and correction of left cubitus valgus.

10. Radiographic union of left lateral condyle and correction of cubitus valgus is achieved (lateral view).

II.After $2 \frac{1}{2}$ months follow up.

12. Radiographic results after $31 / 2$ months.
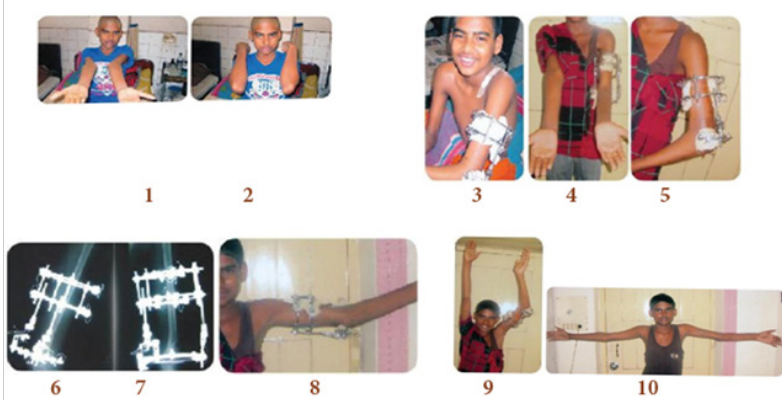

9
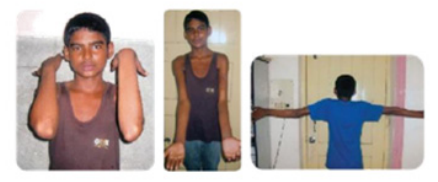

11

12

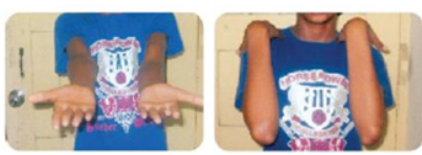

Figure 3 Case 2.

I. 14 years old boy left cubitus valgus elbow in extension.

2. Flexion position before surgery.

3. Smilling patient with Ilizarov apparatus in the left humerus.

4. Full correction of left cubitus valgus with llizarov apparatus in situ after I month follow up.

5. Lateral view of left humerus with llizarov apparatus after one month follow up.

6. Radiographic view of opening wedge osteotomy with placement of hinges over the CORA (Center of rotation of angulation) after 2 months follow up.

7. Patient is doing exercises with llizarov apparatus. 
8. Patient can continue exercises with the llizarov apparatus.

9. Clinical appearance of the patient after 3 months follow up (front view in extension of elbow).

10.Clinical appearance of the patient after 4 months follow up.

I I. Clinical appearance of the patient after 3 months follow up (front view).

12. Clinical appearance of the patient after 3 months follow up (back view in abduction).

13. Clinical appearance of the patient after 3 months follow up (back view in extension of elbow).

14. Final radiographic result after 4 months follow up.

15. Clinical appearance of the patient after 4 months follow up.

16. Clinical appearance of the patient after 4 months follow up.

17. Clinical appearance of the patient after 4 months follow up.

\section{Discussion}

Since most techniques achieve a definitive correction in one stage, further adjustment of the osteotomy is not possible even if needed. The anatomy of the supracondylar region makes its difficult to maintain the correction of any deformity by internal fixation with Kirchumer wire, plates and screws. ${ }^{4,5}$

In our series, we strictly followed the principles of deformity correction proposed by Academician Prof Ilizarov. ${ }^{6-8}$ We did not transpose the ulnar nerve in our four patients and they recover after correction of cubitus valgus due to distraction histoneogenesis. Acute correction can cause a traction injury of the ulnar nerve. The way that we have done the osteotomy and gradual controlled coordinated stretching procedure did not worsen a preexisting tardy ulna palsy, rather it improved the neurological problems.

Different authors have reported hypertrophic scars. ${ }^{4,5,9} \mathrm{We}$ did not encounter this because of mini stab incision required.

The gradual correction of cubitus valgus by distraction osteoneogenesis has other advantages. The position of the distal fragment can be adjusted in all planes until complete consolidation has occured. The elbow does not become stiff because the patient can start their exercises immediately. At the end of distraction period, we must evaluate the upper limb clinically and radiologically. Any additional deformity can be corrected easily. We must mention that most of our patients are teenagers.

\section{Conclusion}

Cubitus valgus deformity of the elbow by distraction osteoneogenesis with an Ilizarov method appears to be efficient, safe, stable, reliable and unique method which avoids unsightly scar formation and loss of range of movement. It has advantages, such as the possibility of further correction during the distraction period and it allows early resumption of daily activities. The Ilizarov frame can be removed as an outdoor basis.

\section{Acknowledgments}

None.

\section{Conflicts of interest}

None.

\section{References}

1. Barrett IR, Bellemore MC, Kwon YM. Cosmetic results of supracondylar osteotomy for correction of cubitus varus. $J$ Pediatr Orthop. 1998;18(4):445-447.

2. Kim HT, Lee Js, Yoo Cl. Management of cubitus varus and valgus. $J$ Bone Joint Surg. 2005;87(4):771-780.

3. Gaddy BC, Manske PR, Pruitt DL, et al. Distal humeral osteotomy for correction of posttraumatic cubitus varus. J Pediatr Orthop. 1994;14(2):214-219.

4. Hasler CC. correction of malunion after pediatric supracondylar elbow fractures: closing wedge osteotomy and external fixation. Eur J Traum. 2003;29(5):309-315.

5. Song HR, Cho SH, Jeong ST, et al. Supracondylar osteotomy with Ilizarov fixation for elbow deformities in adults. J Bone Joint Surg. 1997;79(5):748-752.

6. Ilizarov GA. Clinical application of the tension-stress effect for limb lengthening. Clin Orthop. 1990;250:8-26.

7. Paley D. Osteotomy concepts and frontal plane realignment. In: Paley D (Eds.), Principles of deformity correction, (1st edn), Berlin SpringerVerlag, Germany. 2002;99-154.

8. Herzenberg JE, Waanders NA. Calculating rate and duration of distraction for deformity correction with the Ilizarov technique. Orthop Clin North. 1991;22(4):601-611.

9. Bari MM. A color atlas of limb lengthening surgical reconstruction and deformity correction by Ilizarov technique. 2013;330-333. 